\title{
Condutas hemoterápicas da equipe de enfermagem diante de pacientes oncológicos hemotransfundidos
}

\author{
Hemotherapeutic behaviors of the nursing team in the face of blood transfused cancer \\ patients
}

\section{Comportamientos hemoterapéuticos del equipo de enfermería frente a pacientes con cáncer transfundido con sangre}

Andriellen Rabelo Carvalho ${ }^{1 *}$, Tauane Mirele dos Santos ${ }^{1}$, Maria Rosa Santos Lopes ${ }^{1}$, Gecia Raquel Santos Barreto ${ }^{1}$, Fernanda Aguiar de Aragão Dias ${ }^{1}$, Poliana Elma Santos Jurubeba ${ }^{1}$, Taynara Silva dos Anjos ${ }^{1}$, Paula Caroline Bezerra Rocha ${ }^{1}$, Ruth Cristini Torres ${ }^{1}$, Ana Fátima Souza Melo de Andrade ${ }^{1}$.

\section{RESUMO}

Objetivo: Verificar o conhecimento da equipe de Enfermagem sobre hemoterapia e as reações transfusionais. Métodos: Trata-se de uma pesquisa descritiva, explicativa com abordagem quali-quantitativa, realizada em um hemocentro de Sergipe. Para a realização da coleta de dados foi utilizado um questionário, aplicado aos enfermeiros, técnicos e aos pacientes oncológicos. Os dados foram distribuídos em frequências absoluta e relativa e expressos em gráficos e tabelas analisados por meio dos programas Statistical Package For the Social Sciences for Windows (SPSS) versão 21 e Microsoft Excel 2010, para a análise qualitativa utilizou-se a técnica de análise de conteúdo. Resultados: Este estudo foi realizado com amostra de 17 profissionais da equipe de enfermagem e 22 pacientes oncológicos, sendo assim os profissionais foram compostos por 9 enfermeiros e 8 técnicos de enfermagem. Foi possível observar que ao serem questionados 18 (75\%) pacientes relataram já terem apresentado reação transfusional e $4(25 \%)$ afirmaram que a equipe de enfermagem não orienta sobre o procedimento. Conclusão: Conclui-se que os profissionais entrevistados, demostravam pouca capacitação, principalmente referindo-se as reações transfusionais. Entretanto, são interessados na aquisição de conhecimento para melhorar o atendimento dos pacientes no processo transfusional.

Palavras-chave: Cuidados de enfermagem, Transfusão, Oncologia, Reações transfusionais.

\begin{abstract}
Objective: To verify the knowledge of the Nursing team about hemotherapy and transfusion reactions. Methods: This is a descriptive, explanatory research with a qualitative and quantitative approach, carried out in a blood center in Sergipe. To perform the data collection, a questionnaire was used, applied to nurses, technicians and cancer patients. The data were distributed in absolute and relative frequencies and expressed in graphs and tables analyzed using the Statistical Package For the Social Sciences for Windows - SPSS version 21 and Microsoft Excel 2010 programs, for the qualitative analysis the content analysis technique was used. Results: This study was conducted with a sample of 17 professionals from the nursing team and 22 cancer patients, so the professionals were composed of 9 nurses and 8 nursing technicians. It was possible to observe that when questioned $18(75 \%)$ patients reported having already had a transfusion reaction and 4 (25\%) stated that the nursing team does not advise on the procedure. Conclusion: It is concluded that the professionals interviewed, showed little training, mainly referring to transfusion reactions. However, they are interested in acquiring knowledge to improve patient care in the transfusion process.
\end{abstract}

Keywords: Nursing care, Transfusion, Oncology, Transfusion reactions.

${ }^{1}$ Centro Universitário Estácio, Aracaju - SE. *E-mail: enfermeiradricar2@gmail.com 


\section{RESUMEN}

Objetivo: Verificar el conocimiento del equipo de Enfermería sobre las reacciones de hemoterapia y transfusión. Métodos: Se trata de una investigación descriptiva, explicativa con enfoque cualitativo y cuantitativo, realizada en un centro de sangre en Sergipe. Para realizar la recolección de datos se utilizó un cuestionario que se aplicó a enfermeros, técnicos y pacientes oncológicos. Los datos se distribuyeron en frecuencias absolutas y relativas y se expresaron en gráficos y tablas analizados mediante los programas Statistical Package For the Social Sciences para Windows - SPSS versión 21 y Microsoft Excel 2010, para el análisis cualitativo se utilizó la técnica de análisis de contenido. Resultados: Este estudio se realizó con una muestra de 17 profesionales del equipo de enfermería y 22 pacientes oncológicos, por lo que los profesionales estuvieron compuestos por 9 enfermeros y 8 técnicos de enfermería. Se pudo observar que al ser interrogados $18(75 \%)$ pacientes refirieron haber tenido ya reacción transfusional y $4(25 \%)$ manifestaron que el equipo de enfermería no asesora sobre el procedimiento. Conclusión: Se concluye que los profesionales entrevistados, mostraron poca formación, principalmente en lo que se refiere a reacciones transfusionales. Sin embargo, están interesados en adquirir conocimientos para mejorar la atención al paciente en el proceso de transfusión.

Palabras clave: Cuidado de enfermera, Transfusión, Oncología, Reacciones de transfusión.

\section{INTRODUÇÃO}

O câncer é considerado como um problema na saúde pública mundial e está relacionado com o aumento da mortalidade, em virtude das mudanças acontecidas nos padrões demográficos, como o envelhecimento populacional e dos avanços tecnológicos que interfere de forma negativa no crescimento das doenças crônicas. Logo, a equipe de Enfermagem precisa desenvolver técnicas direcionadas para uma assistência de qualidade através de ações de prevenção, promoção, diagnóstico e tratamento (LINS FG, et al., 2018).

Apesar disso, o ato de cuidar e as ações de enfermagem devem estar voltadas ao respeito, a empatia, e a humanização, para que todos tenham uma boa resposta no enfrentamento do problema e na realização do tratamento dos pacientes oncológicos hemotransfundidos. Pois, os profissionais de saúde estão em contato constante com esses pacientes, participando de todas as etapas do processo do desenvolvimento da doença, do início e término do tratamento. Visto que, a segurança do paciente é consequência das ações que a equipe de enfermagem deverá abordar, além de ser uma das metas de qualidade para o serviço diante da terapia transfusional (GONÇALVES MM, et al., 2018).

Para tanto, se faz necessario que o enfermeiro, junto à sua equipe, deverá ter segurança na prática, considerando os padrões de qualidade e princípios éticos, tendo como responsabilidade uma atenção maior ao paciente oncologicos. Todavia, o enfermeiro deve possuir competência e habilidade para avaliar, prevenir e decidir quais condutas serão mais adequadas para o quadro do paciente (SILVA GEM, et al., 2015).

Desse modo, é importante destacar que os pacientes oncológicos necessitam de um atendimento de qualidade e um cuidado minucioso diante da terapia transfusional. Sabe-se que os indivíduos portadores de câncer e seus familiares sofrem muitas modificações na vida diária, na qual o conhecimento da equipe multiprofissional é de grande importância na qualidade da assistência prestada (MELO WS, et al., 2015).

É evidente que as dúvidas e as dificuldades sobre à hemoterapia, são frequentes em relação à transfusão de sangue. Pois, à terapia transfusional é um procedimento invasivo e bastante utilizado no tratamento de pacientes com câncer (FREITAS ACS, et al., 2014).

Entretanto, a equipe responsável pela hemoterapia deverá adotar condutas com propósito de assegurar um procedimento transfusional de qualidade e objetivando minimizar casos de reações, que são mais evidenciadas no decorrer das transfusões. Com isso, a equipe de enfermagem tem um papel essencial em todo o processo de coleta de sangue, armazenamento, monitorização do paciente, e a prática de hemotransfusão. Porém, são procedimentos que podem acarretar em eventos adversos e intercorrências, onde o enfermeiro necessita buscar constantemente atualização e conhecimento, visando promover o bemestar para este paciente e prevenir riscos (CARVALHO CMS, et al., 2016). 
Diante deste cenário, surgem as questões norteadoras do estudo: Quais as condutas de enfermagem durante o processo transfusional? Os profissionais de enfermagem tem conhecimento sobre a hemoterapia e as reações transfucionais?

O presente estudo tem como hipótese que os pacientes diagnosticados com câncer, submetidos ao tratamento oncológico, apresentam uma grande necessidade de hemotransfusão, mas nem sempre a equipe está preparada para prestar a assistência adequada.

O estudo justifica-se pela importância do conhecimento da equipe como dos pacientes oncológicos e dos benefícios da hemoterapia, já que muitas vezes são submetidos ao tratamento sem a adequada compreensão de sua finalidade. Vale ressaltar que a motivação para esta pesquisa se deu pela percepção das pesquisadoras durante a vivência de estágio extracurricular e o contato com familiares e amigos que possuem uma carência de conhecimento sobre a hemoterapia, da sua importância e os benefícios para pacientes oncológicos.

Acredita-se que esta pesquisa possa contribuir para subsidiar uma assistência humanizada, eficaz e uma técnica correta dos profissionais de enfermagem na transfusão de sangue. Assim, objetiva-se verificar o conhecimento da equipe de Enfermagem sobre hemoterapia e as reações transfucionais e identificar quais as condutas de enfermagem durante o processo transfuncional.

\section{MÉTODOS}

Trata-se de uma pesquisa descritiva, explicativa com abordagem quali-quantitativa. A amostra foi composta por enfermeiros e pacientes oncológicos submetidos à hemotransfusão em um Hemocentro de Sergipe.

Foram incluídos no estudo os enfermeiros do serviço de Hemoterapia e os pacientes oncológicos submetidos à transfusão sanguínea, maiores de 18 anos, que aceitaram participar da pesquisa. Foram excluídos os enfermeiros que estavam de férias ou licença e os pacientes que tiveram incapacidade de compreender os questionamentos.

Para a realização da coleta de dados foi utilizado um questionário, que foi aplicado aos enfermeiros, técnicos e aos pacientes oncológicos. O tratamento dos dados ocorreu mediante a técnica de análise de conteúdo, tal como citou Bardin L (2011), e tiveram como regras as questões referentes à hemoterapia e as reações transfusionais, com questões abertas e subjetivas pertinentes à prática transfusional e às suas abordagens na atuação da equipe e às dúvidas e expectativas dos pacientes diante o procedimento.

A coleta de dados foi respondida pela equipe e pacientes oncológicos, além da coleta de dados secundária dos prontuários e evidências de reações transfusionais. A coleta ocorreu em horários e turnos alternados, considerando a disponibilidade das pesquisadoras e do cenário do estudo.

$\mathrm{O}$ instrumento usado para coletar as informações dos profissionais foi composto por 02 partes: a primeira objetivou caracterizar a amostra, coletando dados como sexo, faixa etária, categoria profissional, tempo de atuação na assistência realizada no hemocentro e qualificação profissional; a segunda parte do instrumento foi constituída por dados relativos à prática transfusional e sua abordagem na atuação do profissional de enfermagem.

Já outro instrumento usado para coletar as informações dos pacientes oncológicos foi composto por 1 parte onde objetivou caracterizar a amostra, coletando dados como sexo, faixa etária, categoria profissional e foram coletados dados relativos ao paciente sobre a terapia transfusional, se houve alguma reação transfusional e se eles têm conhecimento sobre o procedimento.

A coleta de dados teve início após a leitura e a assinatura de um Termo de Consentimento Livre e Esclarecido (TCLE) por todos os pesquisados no setor, nos turnos de trabalho, nos meses de setembro e novembro de 2019. Os sujeitos da pesquisa foram orientados a responder na presença dos pesquisadores, 0 que ajudou uma coleta de dados sem interferência externas e internas, dando fidelidade às respostas coletadas. 
Os dados foram analisados por meio dos programas Statistical Package For the Social Sciences for Windows (SPSS_versão 21 e Microsoft Excel 2010 e foram distribuídos em frequências absoluta e relativa e expressos em gráficos e tabelas. Para a análise qualitativa utilizou-se a técnica de análise de conteúdo (BARDIN L, 2011). Nos casos em que foi necessária a descrição das falas dos sujeitos nos resultados e na discussão, foram identificados pela letra PS (Profissionais de Saúde) seguido de um número de ordem 0-10.

Cumpre ressaltar que as pesquisadoras respeitaram os aspectos éticos e legais da pesquisa envolvendo seres humanos, preconizados pela Resolução oㅜ 466/12 do Conselho Nacional de Saúde, principalmente 0 princípio ético da autonomia, sobretudo o que se refere ao termo de consentimento livre esclarecido (TCLE). A pesquisa foi submetida à apreciação do Comitê de Ética e Pesquisa (CEP) da Estácio de Sergipe, aprovada pelo parecer no 3592355, CAAE 19479919.4.0000.8079, por meio da Plataforma Brasil. Os dados coletados e analisados serão mantidos por cinco anos na posse das pesquisadoras para eventuais comprovações.

\section{RESULTADOS E DISCUSSÃO}

Esse estudo foi realizando com amostra de 17 profissionais da equipe de enfermagem e 22 pacientes oncológicos, sendo assim os profissionais foram compostos por 9 enfermeiros e 8 técnicos de enfermagem. Houve predomínio do sexo feminino de 8 (89\%), com idade média de 40 anos. Quanto a caracterização ao tempo que atuam na assistência, houve uma variação de 6 meses a 7 anos (90\%) e de 16 a 27 anos (10\%), e é saliente destacar que nenhum dos enfermeiros estudados possuem pós-graduação na área de hemoterapia. Quanto a caracterização dos pacientes participantes a amostra foi composta por $22(100 \%)$ pacientes oncológicos onde houve predomínio de idades entre 35 (25,4\%) à 67 (74,6\%) anos.

Com relação ao processo transfusional, 18 (75\%) pacientes já haviam apresentado reações transfusionais e tinham conhecimento sobre como identificar uma reação e 4 (25\%) afirmaram não apresentar conhecimento diante tais reações transfusionais e quais cuidados são necessários, além disso, demostraram pouco conhecimento sobre a importância do processo transfusional, estes dados podem ser observados na Tabela 1.

Foi possível observar que ao serem questionados 18 (75\%) pacientes relataram já terem apresentado reação transfusional e $4(25 \%)$ afirmou e que a equipe de enfermagem não orienta sobre o procedimento. Logo, os cuidados que os profissionais de enfermagem devem ter antes e durante o procedimento transfusional é primordial, pois constatou-se que uma parcela dos pesquisados não demostraram conhecimento suficiente para realização do procedimento ou atuam a pouco tempo no setor.

Tabela 1 - Distribuição dos pesquisados de acordo com idade, sexo e conhecimento sobre reações transfusionais.

\begin{tabular}{ccc}
\hline Características & N & $\%$ \\
\hline Idade & & $38,2 \%$ \\
\hline $35-40$ & 8 & $39,9 \%$ \\
$41-50$ & 9 & $21,9 \%$ \\
$51-67$ & 5 & \\
\hline Sexo & & $36,4 \%$ \\
\hline Masculino & 8 & $63,6 \%$ \\
\hline Feminino & 14 & $78,5 \%$ \\
\hline Conhecimento sobre reação transfusional & & 21,8 \\
\hline Sim & 15 & \\
\hline
\end{tabular}

Fonte: Carvalho AR, et al., 2020. 
Os resultados do estudo mostram que o conhecimento teórico e prático deve caminhar junto do embasamento para a realização do procedimento transfusional, onde é necessário identificar principalmente tipos de reações, nas quais a atuação do profissional tende a buscar a melhoria dos pacientes, além de garantir a segurança transfusional e a diminuição de novos casos de reações (AMARAL JMS, et al., 2016).

Conforme Luz KR, et al. (2016), as condutas que a equipe de enfermagem presta aos pacientes oncológicos são muito importantes, pois eles precisam de atenção diferenciada para lidar continuadamente com eles e suas famílias.

Exemplo disso, é a hemoterapia que auxilia na melhoria do quadro clínico e da qualidade de vida dos pacientes em tratamento. Logo, se faz necessário identificar o conhecimento da equipe que irá prestar os cuidados a estes pacientes, ou seja, é necessário delinear as condutas de enfermagem diante de pacientes oncológicos hemotransfundidos.

Com relação a segurança na execução das técnicas, deverá respeitar os padrões de qualidade e os princípios éticos, tendo como responsabilidade uma atenção diferenciada ao paciente. Desse modo, o enfermeiro deve possuir competência e habilidade para avaliar, prevenir e decidir quais condutas serão as mais adequadas aos pacientes e seus familiares (SILVA JDB, et al., 2018).

Foi possível observar neste estudo que os profissionais de saúde ao serem questionados sobre as condutas adequadas para realizar um melhor atendimento aos pacientes oncológicos, submetidos a transfusão sanguínea, eles demostram dificuldades enfrentadas no serviço de hemoterapia frente a transfusão.

Evidenciou-se que a maioria da equipe acredita ser necessária a realização de treinamentos em relação a importância do uso de Equipamentos de Proteção Individuais (EPl's) e registros relacionados à transfusão, assim como os cuidados necessários na pré-transfusão, tais como: como data, hora, sinais vitais no início e no término do procedimento, origem e identificação das bolsas de hemocomponentes, identificação do profissional responsável e o registro de reação transfusional.

Porém, no presente estudo, foi possível observar que alguns dos profissionais declararam ter conhecimento e foram destacados alguns critérios importantes durante todo o procedimento pré transfusional, onde cerca de $33,(52 \%)$ relatou que o critério importante é a verificação dos sinais vitais, como também conferir os dados, os tipos sanguíneos e registrar, e destes, $(25,5 \%)$ relatou sobre os sinais vitais, a questão da orientação ao paciente e o uso adequado dos EPI's, e outras variações e critérios onde estes dados podem ser observados na Tabela 2.

Segundo Freitas JF, et al. (2014), relata que nem sempre as condutas assistenciais dos profissionais de saúde são adequadas frente às reações transfusionais e que a insegurança na técnica poderá ocasionar vários riscos para o receptor, como evidenciado neste estudo.

Do mesmo modo, a transfusão de sangue aborda vários benefícios para saúde e riscos ao receptor, pois as reações transfusionais muitas das vezes ocorrem principalmente em indivíduos com câncer, devido a sua fragilidade e histórico, ou devido a erros durante a transfusão (GRANDI JL, et al., 2018).

Portanto, a transfusão equivale a administração do sangue em um indivíduo, que se não houver uma técnica segura poderá representar vários riscos. Porém, existem várias maneiras de minimizar possíveis danos, tais como estratégias clínicas através de medicamentos ou equipamentos para tratar distúrbios na coagulação do sangue.

Sendo que, é de suma importância que o enfermeiro esteja frente as transfusões, porém, ele precisa estar capacitado e sempre alerta para possíveis sinais e sintomas que o paciente poderá apresentar durante à transfusão. Em meio a todo esse processo que envolve o tecido sanguíneo, vale destacar como é essencial os cuidados que a enfermagem deve possuir no processo de transfusão (CHEREM EO, et al., 2016). 
Tabela 2 - Critérios relatado importante pela equipe de enfermagem durante o procedimento pré transfusional.

\begin{tabular}{ccc}
\hline Critérios transfusionais & $\mathbf{N}$ & $\%$ \\
\hline $\begin{array}{c}\text { Sinais vitais; } \\
\text { Sinais vitais, orientar o paciente, } \\
\text { uso adequado dos EPI's; } \\
\begin{array}{c}\text { Sinais vitais, confirmar a } \\
\text { transfusão; }\end{array}\end{array}$ & 2 & $10,5 \%$ \\
$\begin{array}{c}\text { Sinais vitais, escolher o equipo } \\
\quad \text { adequado; }\end{array}$ & 2 & $25,5 \%$ \\
$\begin{array}{c}\text { Sinais vitais, conferir dados, tipo } \\
\text { sanguíneo, registrar; } \\
\text { Uso adequado dos EPI's; } \\
\text { Registrar, checar a etiqueta da } \\
\text { bolsa. }\end{array}$ & 1 & $10,5 \%$ \\
\hline
\end{tabular}

Fonte: Carvalho AR, et al., 2020.

Um dos dados importantes, diante do resultado obtido no estudo, foi a respeito dos hemocomponentes mais utilizados na terapia transfusional, o concentrado de hemácias $(\mathrm{CH})$, cujo número de bolsas administradas correspondeu a $85 \%$ do total de unidades transfundidas, ou seja, cerca de $80 \%$ dos pacientes submetidos ao procedimento transfusional eram pacientes oncológicos e que possuem mais facilidade de apresentar uma reação transfusional, onde se faz necessário ter uma atenção maior da equipe durante o procedimento.

Para tanto, é recomendado que a equipe esteja ciente das indicações clínicas e contraindicações para a hemoterapia e das possíveis condições de saúde no paciente oncológico, ou seja, tem como metas promover e prevenir riscos que possam gerar reações transfusionais, por exemplo, o uso do concentrado de hemácias (CH) simples (OLIVEIRA RM, et al., 2014).

Portando, a equipe deverá registrar todas ações e reações durante o procedimento no prontuário, bem como, ao término do procedimento, deverá verificar e comparar com os valores dos sinais vitais registrados anteriormente, pois é um dos meios de identificação de possíveis reações.

Outras medidas utilizadas na prevenção de possíveis reações dadas pela literatura, como foi observado por Mattia D, et al. (2016), são os registros relacionados à transfusão, como data, hora, sinais vitais no início e no término do procedimento, origem e identificação das bolsas, hemocomponentes, identificação do profissional responsável e o registro de reação transfusional. Além da verificação e registro dos sinais vitais como a temperatura, frequência respiratória, pressão arterial e frequência cardíaca.

Certamente as reações transfusionais podem ocasionar possíveis alterações na resposta imunológica do indivíduo, tais como a infiltração, inflamação, infecção, duração da terapia medicamentosa e a infusão de hemocomponentes. Estes fatores determinarão qual foi o tipo de reação adversa transfusional que o paciente poderá desenvolver e qual será a melhor conduta terapêutica indicada para o caso (SILVA AT, et al., 2018).

Os resultados mostram que se faz necessário o conhecimento acerca dos tipos de reações transfusionais sendo elas reações imediatas, a exemplo, as reações alérgicas, anafiláticas, hipotensivas e a sobrecarga circulatória associada à transfusão e as reações tardias, como a reação hemolítica e a púrpura póstransfusão. Demonstrando, assim, a importância do cuidado e da identificação de cada paciente, bem como dos SSVV durante todo o procedimento, pois a ausência de identificação pode resultar vários problemas, semelhantes aos evidenciados.

Com relação as condutas que a equipe deverá realizar em casos de reação transfusional, onde cerca de $48,9 \%$ dos entrevistados afirmaram conhecer os riscos associados e declaram preparação para realizar a assistência necessária e 51,1 \% já presenciaram reações transfusionais e se sentem capacitados. Na Tabela 3 está descrito os dados a serem observados no que diz respeito às condutas de enfermagem diante uma reação transfusional. 
Tabela 3 - Conhecimento da equipe de enfermagem sobre as condutas apontados como corretas diante de uma reação transfusional.

\begin{tabular}{ccc}
\hline Condutas & $\mathbf{N}$ & $\%$ \\
\hline $\begin{array}{c}\text { Interromper a transfusão e manter o acesso; } \\
\begin{array}{c}\text { Comunicar ao médico, interromper a transfusão, manter o acesso e } \\
\text { verificar os sinais vitais; }\end{array}\end{array}$ & 4 & $15,9 \%$ \\
\hline $\begin{array}{c}\text { Verificar os sinais vitais, manter o acesso e monitorar o paciente; } \\
\text { Comunicar ao banco de sangue e registrar no livro de reações } \\
\text { transfusionais. }\end{array}$ & 5 & $35,8 \%$ \\
\hline
\end{tabular}

Fonte: Carvalho AR, et al., 2020.

Foi possível observar neste estudo a prevalência do índice de que os profissionais de saúde estão em contato constante com estes pacientes, onde estão participando de todas as etapas do processo do desenvolvimento do câncer e do início e término do tratamento oncológico. Diante da transfusão, a equipe deverá ser capaz de reconhecer os sinais e sintomas da doença em que o paciente apresenta e entender como ocorrem as reações transfusionais.

Diante dos resultados obtidos na Tabela 4, apresentando a distribuição dos participantes que, em algum momento, já presenciaram reações transfusionais, foram encontrados $n^{\circ} 17(100 \%)$ dos entrevistados que deram a resposta sobre os sinais e sintomas que aconteceram em pacientes oncológicos no ano de 2019.

Tabela 4 - Participantes que presenciaram reações transfusionais e os sinais e sintomas que mais acometem.

\begin{tabular}{lcc}
\hline Questionário & N (17) & $\%$ \\
\hline Presenciou alguma reação transfusional & & $79 \%$ \\
\hline Sim & 12 & \\
Não & 5 & $62,07 \%$ \\
\hline Sinais e sintomas & & $31,03 \%$ \\
\hline Febre, dor no local do acesso & 13 & $31,03 \%$ \\
Hrurido, calafrios, mal-estar & 5 & $13,79 \%$ \\
Parada cardiorrespiratória & 5 & $62,07 \%$ \\
Alterações dos sinais vitais & 4 & $6,90 \%$ \\
Vômito, sudorese & 13 & \\
\hline
\end{tabular}

Fonte: Carvalho AR, et al., 2020.

O enfermeiro possui uma responsabilidade muito grande ao prestar os cuidados contínuos aos pacientes oncológicos, pois estes necessitam de uma atenção diferenciada, visto que o tratamento oncológico possui muitos efeitos adversos e cuidado diferencial (SALIMENA AMO, et al., 2013).

Diante dos resultados expostos, após a avaliação da interpretação e organização das falas, as informações foram delimitadas e para manter $o$ anonimato dos participantes, os depoimentos foram classificados pelas letras PS (Profissional de Saúde), seguida de números de 01 a 10 (PS 01, PS02).

No princípio da pesquisa os enfermeiros e técnicos destacaram, prioritariamente, as questões sobre a adequação de alguns materiais para compor uma forma mais adequada a sala de emergência e equipamentos melhorando $o$ atendimento e, também, relataram a dificuldade de contato com os pacientes.

[...] Dificuldade na questão da falta de alguns materiais e equipamentos para ajudar no atendimento ao paciente (PS08; PS04; PS06). Ausência de treinamento em serviço de forma sistêmica (PS03; PS05).

Ao considerar que a conduta da equipe de enfermagem possui um papel crucial no atendimento aos pacientes submetidos à hemoterapia, deve-se identificar os obstáculos enfrentados devido à dificuldade de acesso venoso periférico, como também, a aceitação do paciente ao tratamento, entre outras questões. 
É necessário, ainda, que a equipe passe as informações necessárias a estes pacientes sobre os fatores intervenientes e as competências que envolvem o exercício da função. Neste cenário, alguns relatos merecem destaque, conforme exposto abaixo:

\section{[...]poucas informações aos critérios da hemoterapia e sua finalidade (PS01; PS09) [...] relacionamento interpessoal (PS02; PS07; PS10).}

O que foi observado neste estudo e, de acordo com a população, possibilitou uma melhor compreensão do processo hemoterápico e diante dos procedimentos pré, trans. e pós-transfusionais que contribuindo para a melhoria do quadro clínico dos pacientes oncológicos em tratamento e quais são as condutas necessárias. Pois, nesse sentido, a enfermagem desempenha um papel fundamental na hemoterapia, por possibilitar que eles abordem as principais condutas a serem tomadas nas reações transfusionais, tornando a sua presença requerida em todas as etapas da assistência na hemoterapia (FORSTER F, et al., 2018).

\section{CONCLUSÃO}

Foi possível concluir que, frente as condutas de enfermagem durante o processo transfusional e sobre 0 conhecimento da equipe sobre a hemoterapia e das reações transfusionais, a maioria dos entrevistados, demostravam pouca capacitação, principalmente referindo-se as reações transfusionais. Entretanto, transpareceram interessados na aquisição de conhecimento para melhor atendimento dos pacientes. Tendo em vista os argumentos apresentados, se torna imprescindível o desenvolvimento de estratégias voltadas para a educação em saúde contínua visando, assim, o melhor preparo da equipe para um atendimento adequado e de qualidade voltado para pacientes oncológicos submetidos ao processo transfusional, além de ser evidente que o enfermeiro tem papel crucial no cuidado destes pacientes, com a finalidade de reduzir danos e riscos relacionados à ocorrência de uma reação transfusional.

\section{REFERÊNCIAS}

1. AMARAL JHS, et al. Hemoterapia: um desafio no cotidiano da equipe de enfermagem. Revista Enfermagem UFPE, 2016; 10(6): 482- 487.

2. BARDIN L. Análise de conteúdo. 70nd ed. São Paulo, 2011;229p.

3. CARVALHO CMS, et al. Sentimentos de mulheres com diagnóstico de câncer de mama. Revista Enfermagem UFPE, 2016; 10(11): 342-350.

4. CHEREM EO, et al. Cuidado Pós - Transfusional na unidade de terapia intensiva neonatal. Revista Baiana de Enfermagem, 2016;30(4): 1-8.

5. FORSTER F, et al. Percepção dos enfermeiros quanto à assistência de enfermagem no processo transfusional. Revista Enfermagem Foco, 2018; 9(3): 71-75.

6. FREITAS JF, et al. Perfil das reações transfusionais em pacientes pediátricos oncológicos. Revista Enfermagem UFPE, 2014; 8(9): 30-38.

7. FREIRA ACS, et al. Doação de sangue: conhecimento, prática e atitude de acadêmicos de enfermagem de uma instituição do interior do Ceará. Revista Mineira de Enfermagem, 2013; 17(2): 296-303.

8. GRANDI JL, et al. Hemovigilance: the experience of transfusion reaction reporting in a Teaching Hospital. Revista Escola de Enfermagem USP, 2018; 52: 03-33.

9. GOLÇALVES MM, et al. Perfil dos atendimentos a pacientes oncológicos em uma unidade de pronto atendimento. Revista de Enfermagem do Centro-Oeste, 2018; 8: 25-95.

10. JARDIM VLT, et al. Transfusão de sangue - o conhecimento dos profissionais de enfermagem. Revista Enfermagem UFPE, 2014; 8(6): 49-57.

11. LINS FG, et al. Formação dos enfermeiros para o cuidado em oncologia. Revista Enfermagem UFPE, 2018; 12(1): 66-74.

12. LUZ KR, et al. Enfermeiros na atenção oncológica: conhecimento na prática do cuidado. Revista Enfermagem UFPE, 2016; 10(9):69-76.

13. MATTIA D, et al. Cuidados de enfermagem na transfusão de sangue: Um instrumento para monitorização do paciente. Revista Texto Contexto Enfermagem, 2016; 2: 15-26.

14. MELO WS, et al. Caracterização do recém-nascidos hemotransfundidos na unidade de terapia intensiva neonatal. Revista Enfermagem UFPE, 2015; 9(2): 2-9.

15. OLIVEIRA RM, et al. Estratégias para promover segurança do paciente: da identificação dos riscos às práticas baseadas em evidências. Esc. Anna Nery Revista De Enfermagem, 2014; 18(1): 14-92.

16. OLIVEIRA MCL, et al. Sentimentos dos profissionais de enfermagem em relação ao paciente oncológico. Revista Mineira Enfermagem, 2012; 16(1): 91-97. 
17. SALIMENA AMO, et al. Estratégias de enfrentamento usadas por enfermeiros ao cuidar de pacientes oncológicos. Revista Enfermagem UFSM, 2013; 3(1): 8-16.

18. SANTOS SP, et al. Avaliação dos registros de enfermagem em hemoterapia de um hospital geral. Revista Av. Enfermagem, 2013; 11(1): 103-112.

19. SILVA AT, et al. Segurança do paciente e a atuação do enfermeiro em Hospital. Revista Enfermagem UFPE, 2018; 12(6): 31-38.

20. SILVA JDB, et al. As hemotransfusões e atuação do enfermeiro no procedimento alternativo. Braz. J. Surge. Clínica Revista, 2018; 23(1): 100-105.

21. SILVA GEM, et al. Conhecendo os meandros da doação de sangue: implicações para a atuação do enfermeiro na hemoterapia. Revista Brasileira de Enfermagem, 2015; 68(1): 32-39. 